\title{
Inhibition of dipeptidyl peptidase IV protects tacrolimus-induced kidney injury
}

\author{
Sun W Lim ${ }^{1,2}$, Long Jin ${ }^{1,2}$, Shang G Piao ${ }^{1,2}$, Byung H Chung ${ }^{1,3}$ and Chul W Yang ${ }^{1,2,3}$
}

Accumulating evidence shows that a gut-released hormone, the glucagon-like peptide-1 (GLP-1), has not only a glucoselowering effect but also a renoprotective effect against kidney injury. In this study, we investigated whether a dipeptidyl peptidase (DPP) IV inhibitor has a protective effect against tacrolimus-induced renal injury. Rats were treated with tacrolimus ( $1.5 \mathrm{mg} / \mathrm{kg}$, subcutaneously) and the DPP IV inhibitor MK0626 (10 or $20 \mathrm{mg} / \mathrm{kg}$, oral gavage) for 4 weeks. MK0626 treatment attenuated tacrolimus-induced renal dysfunction, tubulointerstitial fibrosis, and arteriolopathy. Moreover, these improvements were accompanied by a reduction in oxidative stress and apoptosis. MK0626 treatment increased the blood level of GLP-1 and the level of its receptor in tissue sections but did not alter the levels of other DPP IV substrates, such as neuropeptide $Y$ and the stromal cell-derived factor-1. These data suggest that DPP IV inhibition has an important role in the renoprotection against tacrolimus-induced nephrotoxicity via antioxidative and antiapoptotic effects and preservation of the GLP-1 system.

Laboratory Investigation (2015) 95, 1174-1185; doi:10.1038/labinvest.2015.93; published online 3 August 2015

Tacrolimus (TAC) is a maintenance immunosuppressant that is widely used in renal transplant recipients. However, it causes considerable chronic allograft dysfunction and allograft failure in these individuals. Experimental studies show that treatment with TAC causes progressive renal failure with striped interstitial fibrosis, tubular atrophy, inflammatory cell infiltration, and hyalinosis of the afferent arterioles. ${ }^{1}$ Although the pathogenesis of TAC-induced nephropathy remains undetermined, this condition is partly related to the direct toxic effect of TAC on renal cells, and oxidative stress has a pivotal role in TAC-induced renal dysfunction and cell damage. $^{2-4}$

Dipeptidyl peptidase (DPP) IV inhibitors represent a new class of antidiabetic agents based on their ability to extend the biological effects of incretin hormones, such as the glucagonlike peptide-1 (GLP-1). ${ }^{5,6}$ A considerable body of research has confirmed that DPP IV inhibitors can prevent beta-cell failure, stimulate insulin release, improve glycemic and hemoglobin A1c control, and decrease triglyceride and free fatty acid levels in diabetic patients. ${ }^{7,8}$ In addition to its glucose-lowering effects, DPP IV inhibitors also ameliorate renal damage caused by ischemia-reperfusion, cisplatin treatment, 5/6 nephrectomy, and diabetic nephropathy. ${ }^{9-12}$
These observations suggest that DPP IV inhibitors can exert renoprotective effects partly by increasing GLP-1 levels. However, it remains unclear whether DPP IV inhibitors have a beneficial effect against TAC-induced renal injury, and whether this effect is associated with increased GLP-1 levels, because DPP IV cleaves other molecules, such as neuropeptide Y (NPY) and the stromal cell-derived factor-1 (SDF-1).

Therefore, we designed this study to assess the systemic effect of a DPP IV inhibitor on TAC-induced renal injury. First, we evaluated whether the DPP IV inhibitor protects against TAC-induced renal dysfunction and tubulointerstitial fibrosis, and whether this protection is associated with antioxidative and antiapoptotic effects. Second, we examined whether TAC induced DPP IV activity, and if so, whether this could be effectively reduced by treatment with a DPP IV inhibitor. Third, we investigated which substrates of DPP IV are associated with its protective properties. Our results clearly showed that DPP IV inhibition effectively protects against TAC-induced renal injury. Moreover, whereas other molecule, eg, NPY and SDF-1, were not significantly changed by TACinduced DPP IV activity, GLP-1 had an important role in renal protection. We expect that the results of our study will provide

\footnotetext{
${ }^{1}$ Convergent Research Consortium for Immunologic Disease, Seoul, Korea; ${ }^{2}$ Transplant Research Center, Seoul, Korea and ${ }^{3}$ Division of Nephrology, Department of Internal Medicine, Seoul St Mary's Hospital, The Catholic University of Korea, Seoul, Korea

Correspondence: Professor CW Yang, MD, PhD, Division of Nephrology, Department of Internal medicine, Seoul St Mary's Hospital, The Catholic University of Korea, 222 Banpo-daero, Seocho-gu, Seoul 137-701, Korea.

E-mail: yangch@catholic.ac.kr
}

Received 23 September 2014; revised 20 April 2015; accepted 13 May 2015 
a rationale for the use of DPP IV inhibitor in renal transplant patients receiving TAC.

\section{MATERIALS AND METHODS Animals and Drugs}

The Animal Care and Use Committee of the Catholic University of Korea approved the experimental protocol (CUMC-2012-0117-02), and all procedures performed in this study were in accordance with ethical guidelines for animal studies. Eight-week-old male Sprague Dawley rats (Charles River Technology, Seoul, Korea) that initially weighed 220$230 \mathrm{~g}$ were housed in cages (Nalge, Rochester, NY, USA) in a controlled-temperature and controlled-light environment at the Catholic University of Korea's animal care facility. The rats received a low-salt diet $(0.05 \%$ sodium chloride, Research Diets, New Brunswick, NJ, USA). TAC (Prograft, Astellas Pharma, Ibaraki, Japan) was diluted in olive oil (Sigma, St Louis, MO, USA) to a final concentration of $1 \mathrm{mg} / \mathrm{ml}$. DPP IV inhibitor MK0626 was kindly supplied by Merk Sharp and Dohme (Kenilworth, NJ, USA) and was diluted in drinking water to a final concentration of 10 or $20 \mathrm{mg} / \mathrm{ml}$.

\section{Experimental Design}

The study was designed to evaluate the effect of MK0626 on TAC-induced renal injury. After acclimatization and a lowsalt diet for 1 week, weight-matched rats were randomized to six groups containing eight rats each and were treated daily with TAC $(1.5 \mathrm{mg} / \mathrm{kg}$, s.c.) or vehicle $(\mathrm{VH}$, olive oil, $1 \mathrm{mg} / \mathrm{ml}$, s.c.) with or without MK0626 (M, 10 and $20 \mathrm{mg} / \mathrm{kg}$, oral gavage) for 4 weeks. Routes and doses of administrating drugs were chosen based on the preliminary study. ${ }^{13,14}$

\section{Basic Protocol}

Rats were pair-fed and their body weight was monitored daily. After the 4-week treatments, systolic blood pressure was recorded using the tail cuff method (Visitech System, Apex, NC, USA). Before euthanasia, animals were housed individually in metabolic cages (Tecniplast, Gazzada, Italy) for the measurement of water intake and urine volume over $24 \mathrm{~h}$. On the following day, animals were anesthetized, and blood samples and tissue specimens were obtained for further analysis. Serum creatinine (Scr) and blood urea nitrogen (BUN) were measured using a quantitative enzyme colormetric method (Stanbio Laboratory, Boerne, TX, USA). Creatinine clearance $(\mathrm{ClCr})$ was calculated using a standard formula from 24-h urine collections and serum. The wholeblood TAC level was measured using LC-MS/MS. ${ }^{15}$

\section{Histopathology}

Histological assessment was defined as the development of tubulointerstitial fibrosis and arteriolopathy in Trichrome or periodic acid Schiff (PAS)-stained tissue sections as described previously. ${ }^{16,17}$ Briefly, the tissue sections were re-fixed in Bouin's solution for $1 \mathrm{~h}$ at $56^{\circ} \mathrm{C}$ to improve staining quality. Then they were stained in Weigert's iron hematoxylin working solution for 10 min. After a wash in distilled water (DW), the slides were soaked in the working solution of Biebrich scarlet-acid fuchsin and phosphomolybdicphosphotungstic acid solution, one by one. The slides were transferred directly (without rinsing) to aniline blue solution and stained for 5-10 min and then briefly rinsed in DW and $1 \%$ acetic acid solution. After dehydration through 95\% ethyl alcohol, the slides were mounted with resinous mounting medium. The collagen fibers were stained in blue, the nuclei were stained in purple, and the background was stained in red. A finding of tubulointerstitial fibrosis was defined as a matrix-rich expansion of the interstitium with tubular dilatation, tubular atrophy, tubular cast formation, sloughing of tubular epithelial cells, or thickening of the tubular basement membrane. The extent of fibrosis was estimated by counting the percentage of injured area per field using polygon program. Arteriolopathy of the afferent arterioles was manifested by expansion of the cell cytoplasm of terminal arteriolar smooth muscle cells and semiquantitatively evaluated by counting the percentage of juxtaglomerular afferent arterioles with arteriolopathy per total number of juxtaglomerular afferent arterioles available for examination. A minium of 20 fields per section was assessed and graded using color image analyzer (TDI Scope Eye Version 3.0 for Windows, Olympus, Japan). Histopathological analysis was performed in randomly selected cortical fields of sections by a pathologist blinded to the identity of the treatment groups.

\section{Immunohistochemistry}

Immunohistochemistry was performed as described previously. ${ }^{18,19}$ ED-1, osteopontin (OPN), transforming growth factor $\beta 1$ (TGF $\beta 1$ )-inducible gene-h3 ( $\beta$ ig-h3), renin, 8 -OHdG, and GLP-1R were detected in $4-\mu \mathrm{m}$ tissue sections by incubating cells for $12 \mathrm{~h}$ with specific antibodies against kidney injury molecule-1 (KIM-1; Abcam, Cambridge, UK), neutrophil gelatinase-associated lipocalin (NGAL; Millipore, Billerica, MA, USA), ED-1 (AbD Serotec, Oxford, UK), OPN (obtained from the Developmental Studies hybridoma bank, University of Iowa, Iowa City, IA, USA), $\beta$ ig-h3 (Proteintech, Chicago, IL, USA), 8-OHdG (JaICA, Shizuoka, Japan), and GLP-1R (Abcam) at $4{ }^{\circ} \mathrm{C}$. The KIM-1-, NGAL-, and $\beta$ ig-h3stained tissue sections were counted in 20 different fields in each section at $\times 200$ magnification using color image analyzer (TDI Scope Eye Version 3.0 for Windows).

\section{Immunoblot Analysis}

Immunoblot analysis was performed as described previously ${ }^{18,20}$ Using tissue lysates from the renal cortex, e-cardherin, $\alpha$-smooth muscle actin ( $\alpha$-SMA), TGF $\beta 1$, p-eNOS, t-eNOS, Bcl-2, Bax, Bim, active caspase-3, manganese superoxide dismutase (MnSOD), heme oxygenase-1 (HO-1), HO-2, GLP-1R, and $\beta$-actin were detected by incubating for $12 \mathrm{~h}$ with specific antibodies against e-cadherin (BD Biosciences, San Jose, CA, USA), $\alpha$-SMA (Sigma), TGF $\beta 1$ (R\&D Systems, Minneapolis, MN, USA), p-eNOS (Cell 
Table 1 The effect of MK0626 on TAC-induced renal dysfunction

\begin{tabular}{|c|c|c|c|c|c|c|}
\hline & $\mathrm{VH}(n=8)$ & $\mathrm{VH}+\mathrm{M} 10(n=8)$ & $\mathrm{VH}+\mathrm{M} 20(n=8)$ & TAC $(n=8)$ & $\mathrm{TAC}+\mathrm{M} 10(n=8)$ & $\mathrm{TAC}+\mathrm{M} 20(n=8)$ \\
\hline BW (g) & $268 \pm 5$ & $270 \pm 3$ & $270 \pm 4$ & $279 \pm 6$ & $273 \pm 3$ & $269 \pm 4$ \\
\hline Water intake (ml/day) & $23 \pm 4$ & $19 \pm 2$ & $21 \pm 3$ & $28 \pm 6$ & $26 \pm 3$ & $30 \pm 7$ \\
\hline $\mathrm{SBP}(\mathrm{mm} \mathrm{Hg})$ & $131 \pm 1$ & $128 \pm 1$ & $134 \pm 1$ & $132 \pm 2$ & $133 \pm 1$ & $132 \pm 3$ \\
\hline BUN (mg/dl) & $14 \pm 2$ & $12 \pm 0.4$ & $12 \pm 2$ & $35 \pm 4^{\mathrm{a}-\mathrm{c}}$ & $24 \pm 1^{\mathrm{a}-\mathrm{d}}$ & $24 \pm 2^{\mathrm{a}-\mathrm{d}}$ \\
\hline $\mathrm{ClCr}(\mathrm{mL} / \mathrm{min} / 100 \mathrm{~g})$ & $0.79 \pm 0.07$ & $0.82 \pm 0.03$ & $0.87 \pm 0.08$ & $0.45 \pm 0.04^{\mathrm{a}-\mathrm{c}}$ & $0.56 \pm 0.03^{\mathrm{a}-\mathrm{d}}$ & $0.56 \pm 0.03^{a-d}$ \\
\hline KIM-1 (\%) & $0.62 \pm 0.14$ & $0.63 \pm 0.09$ & $0.63 \pm 0.05$ & $7.42 \pm 0.69^{a-c}$ & $4.74 \pm 0.84^{\mathrm{a}-\mathrm{d}}$ & $2.34 \pm 0.40^{a-e}$ \\
\hline NGAL (\%) & $0.01 \pm 0.01$ & $0.03 \pm 0.09$ & $0.03 \pm 0.01$ & $0.13 \pm 0.03^{a-c}$ & $0.05 \pm 0.02^{a-d}$ & $0.04 \pm 0.01^{a-d}$ \\
\hline TAC level (ng/ml) & - & - & - & $7.5 \pm 0.9$ & $7.5 \pm 0.7$ & $7.9 \pm 0.4$ \\
\hline
\end{tabular}

BUN, blood urea nitrogen; BW, body weight; ClCr, creatinine clearance; M10 and M20, 10 and $20 \mathrm{mg} / \mathrm{kg}$ of MK0626; SBP, systolic blood pressure; Scr, serum creatinine; TAC, tacrolimus; UV, urine volume; VH, vehicle. TAC level, trough blood level of TAC. Values are means \pm s.e. ${ }^{\mathrm{a}} P<0.05 \mathrm{vs} \mathrm{VH}$, ${ }^{\mathrm{b}} \mathrm{P}<0.05 \mathrm{vs} \mathrm{VH}+\mathrm{M} 10$, ${ }^{\mathrm{c}} P<0.05$ vs VH $+\mathrm{M} 20,{ }^{\mathrm{d}} P<0.05$ vs $\mathrm{TAC},{ }^{\mathrm{e}} P<0.05$ vs $\mathrm{TAC}+\mathrm{M} 10$.

Signaling, Beverly, MA, USA), t-eNOS (Cell Signaling), Bcl-2 (Santa Cruz Biotechnology), Bax (Delta Biolabs, Gilroy, CA, USA), Bim (Cell signaling), active caspase-3 (Millipore), MnSOD (Abcam), HO-1 (Enzo Life Sciences, Farmingdale, NY, USA), HO-2 (Enzo Life Science), GLP-1R (Abcam), and $\beta$-actin (Sigma) at $4{ }^{\circ} \mathrm{C}$.

In Situ Terminal Deoxynucleotidyl Transferase-Mediated dUTP-Biotin Nick End Labeling (TUNEL) Assay

Apoptosis in tissue sections or adherent cells on slides was identified using the ApopTag In Situ Apoptosis Detection Kit (Millipore). The number of TUNEL-positive cells was counted in 20 different fields in each section at $\times 200$ magnification.

\section{Measurement of 8-Hydroxy-2'-Deoxyguanosine (8-OHdG)}

Oxidative DNA damage was evaluated based on the level of the DNA adduct 8-OHdG in 24-h urine using a competitive enzyme-linked immunosorbent assay (Cell Biolabs, San Diego, CA, USA).

\section{Measurement of the Levels of DPP IV Activity, Active GLP-1, NPY-1, and SDF-1}

To analyze DPP IV activity in the kidney, renal cortex was homogenated in cold assay buffer $(25 \mathrm{mM}$ Tris- $\mathrm{HCl}, 140 \mathrm{mM}$ $\mathrm{NaCl}, 10 \mathrm{mM} \mathrm{KCl}, \mathrm{pH} 7.5,0.1 \% \mathrm{BSA}$ ) and centrifuged at $20000 \mathrm{~g}$ for $20 \mathrm{~min}$ at $4{ }^{\circ} \mathrm{C}$. To measure plasma DPP IV activity, blood samples were collected into prechilled EDTAtreated tubes, centrifuged at $4{ }^{\circ} \mathrm{C}$, separated, and frozen at $-80^{\circ} \mathrm{C}$. DPP IV activity in the lysate and plasma was measured using the Assay Kit (Millipore). For the quantification of active GLP-1, NPY-1, and SDF-1 in the plasma samples, blood samples were collected into tubes containing a DPP IV inhibitor (50 uM), centrifuged, separated, and frozen at $-80^{\circ} \mathrm{C}$. The level of active GLP-1 (Millipore), NPY-1 (Millipore), and SDF-1 (Sunred, Shanghai, China) were measured using a commercially available ELISA Kit, according to the manufacturer's recommendations.

\section{Statistical Analysis}

The data are expressed as means \pm s.e. of at least three independent experiments. Multiple comparisons between groups were performed by one-way ANOVA with the Bonferroni post hoc test (SPSS software version 19.0; IBM, Armonk, NY, USA). Statistical significance was assumed as $P<0.05$.

\section{RESULTS}

\section{Effect of MK0626 on TAC-Induced Renal Dysfunction}

Table 1 lists the changes in the functional parameters in the experimental group as a result of TAC and MK0626 (M) treatment for 4 weeks. The TAC+M10 or TAC $+\mathrm{M} 20$ groups showed a lower level of Scr and BUN and a higher rate of $\mathrm{ClCr}$ than did the TAC group. We also measured immunoreactivity of renal tubular injury marker, KIM-1 and NGAL on tissue section and enhanced their expression levels by TAC treatment were decreased in TAC+M10 or TAC+M20 groups. MK0626 cotreatment did not affect the trough level of TAC in the whole blood, indicating that a drug interaction did not occur at these doses.

\section{Effect of MK0626 on TAC-Induced Renal Inflammation}

To evaluate the effect of MK0626 on inflammatory cell infiltration and proinflammatory cytokines produced by inflammatory cells, we analyzed the levels of infiltration of ED-1-positive cells and OPN in tissue sections. As shown in Figures 1a and $\mathrm{b}$, the number of ED-1-positive cells was minimal in the $\mathrm{VH}$ and $\mathrm{VH}+\mathrm{M}$ groups. However, TAC treatment significantly increased these numbers, and this 

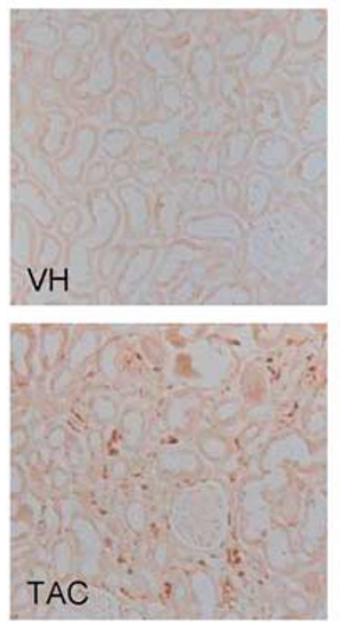

C
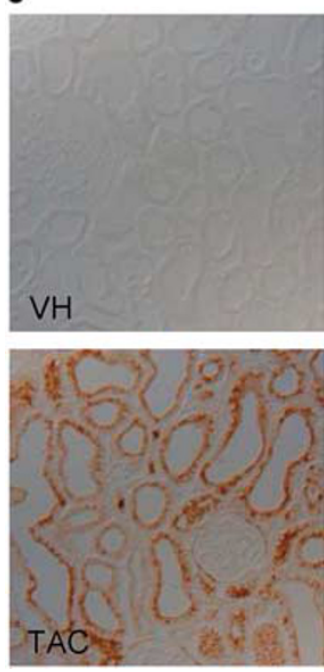
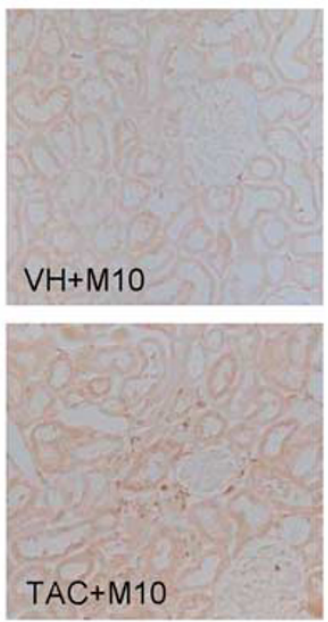

$\mathrm{TAC}+\mathrm{M} 10$
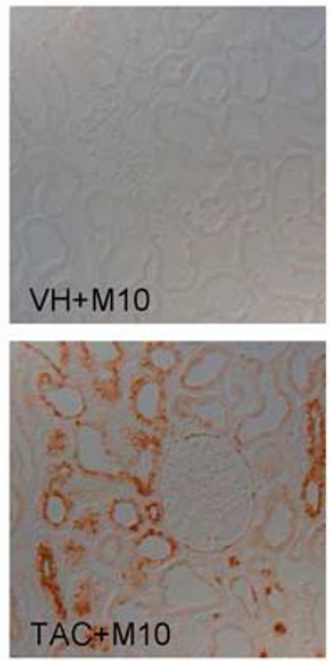
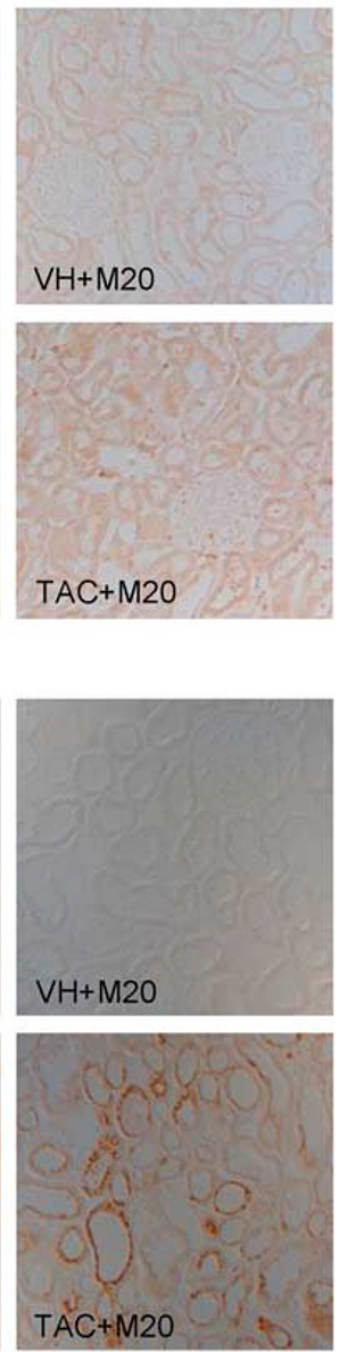

b

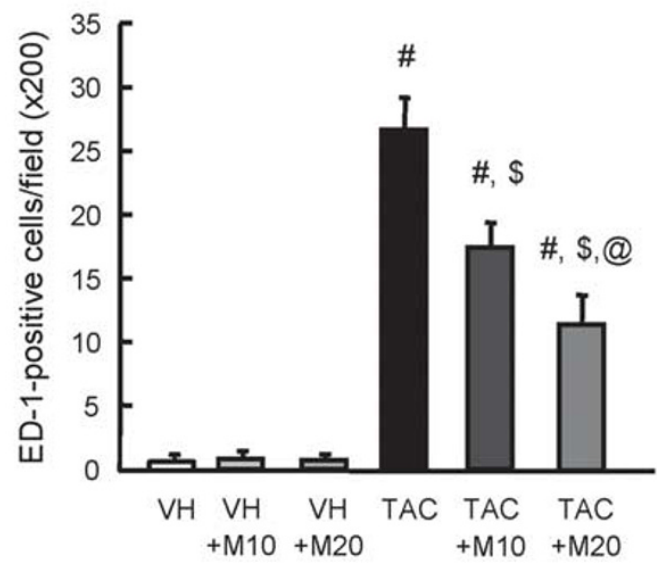

d

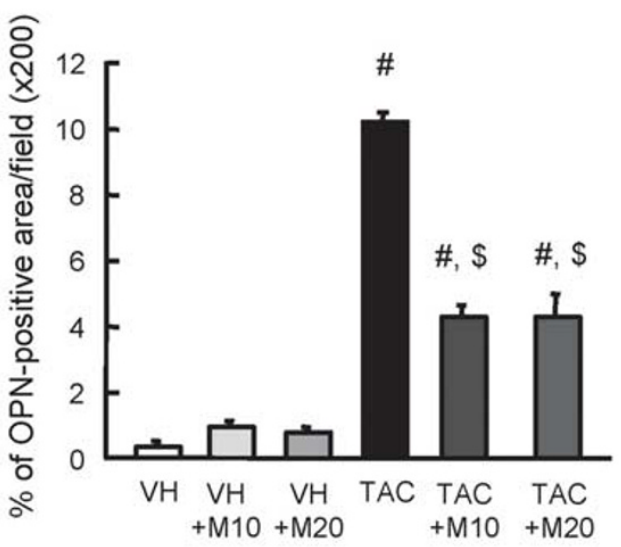

Figure 1 Effect of MK0626 (M) on inflammation during tacrolimus (TAC)-induced renal injury. Immunohistochemistry and its quantitative analysis for ED-1 ( $\mathbf{a}$ and $\mathbf{b}$ ) and osteopontin (OPN) (c and $\mathbf{d}$ ) in the renal cortex from experimental groups. Note that the number of ED-1-positive cells and the intensity of OPN staining are increased in the TAC group and that this increase is inhibited in the TAC + M groups. Magnification, $\times 400 . n=8$ per group. ${ }^{\#} P<0.05$ vs VH; ${ }^{\$} P<0.05$ vs TAC; ${ }^{\circledR} P<0.05$ vs TAC + M 10 .

increase was markedly attenuated by treatment with MK0626. Consistently, strong immunoreactivity for OPN was observed in the TAC group and was attenuated after MK0626 treatment, as shown in Figures 1c and d.

\section{Effect of MK0626 on TAC-Induced Renal Fibrosis}

MK0626 significantly reduced the extensive TAC-induced interstitial fibrosis; however, there was no significant MK0626 dose-dependent effect in this experimental model (Figures 2a and $\mathrm{b}$ ). On a molecular basis, TAC treatment reduced the expression levels of e-cadherin and increased those of $\alpha$-SMA; these levels were restored by treatment with MK0626 (Figures $2 \mathrm{c}$ and $\mathrm{d}$ ). Induction of the TGF $\beta 1$ and of its bioactive form, $\beta \mathrm{ig}$-h 3 , in the TAC group was also attenuated in the TAC+ MK0626 groups, as shown in Figures 2e and f.

\section{Effect of MK0626 on TAC-Induced Arteriolopathy and Endothelial Nitric Oxide Synthase (eNOS) Expression}

TAC treatment decreased the percentage of strong PASpositive cells compared with the $\mathrm{VH}$, whereas this parameter was markedly decreased after treatment with MK0626 compared with TAC treatment (Figures $3 \mathrm{a}$ and $\mathrm{b}$ ). The downregulation of the eNOS protein by TAC treatment was also significantly recovered in the MK0626-treated groups (Figure 3c).

\section{Effect of MK0626 on TAC-Induced Oxidative Stress}

Figures $4 \mathrm{a}$ and $\mathrm{b}$ shows the results of immunohistochemistry and $24-\mathrm{h}$ urinary excretion of $8-\mathrm{OHdG}$, which is a marker of oxidative DNA damage, in the experimental 
a

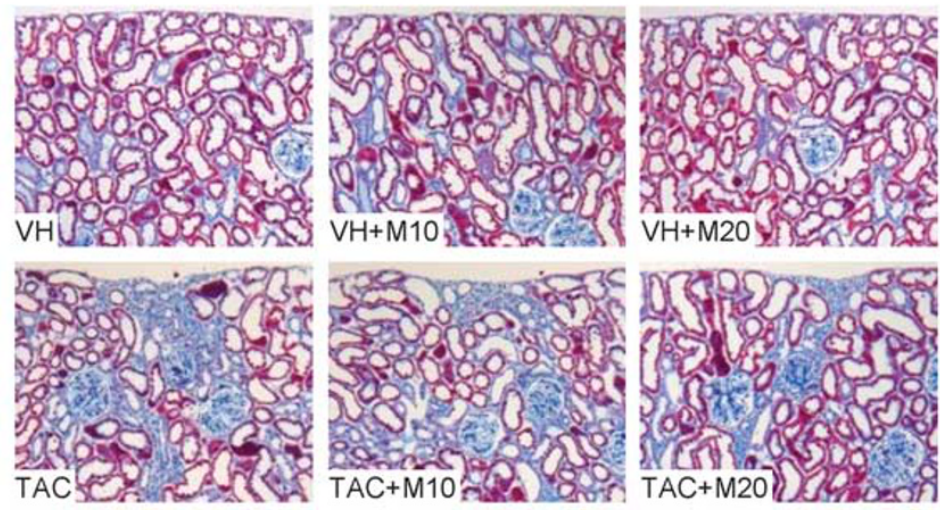

b

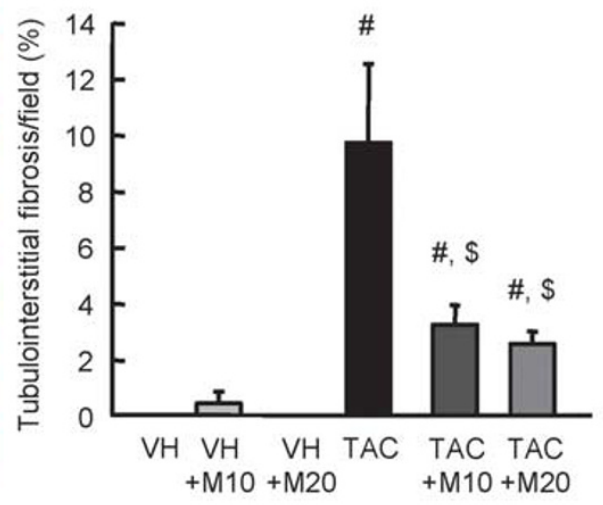

c
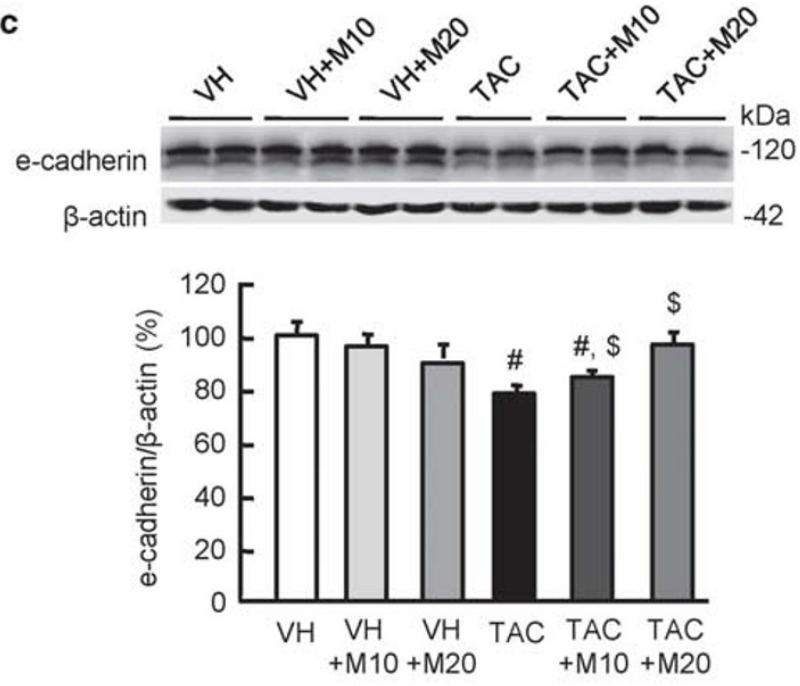

d
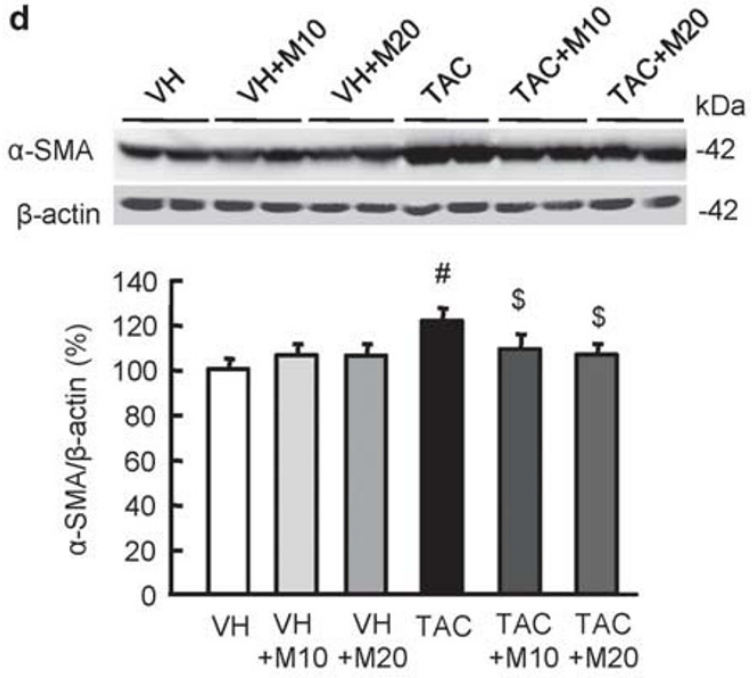

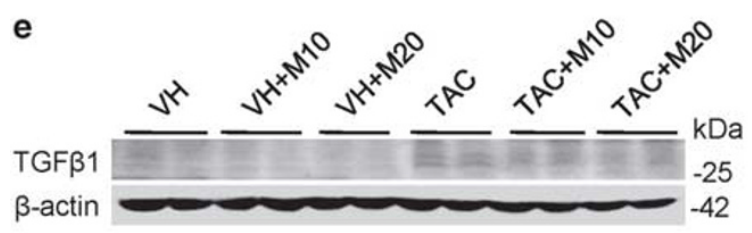

f
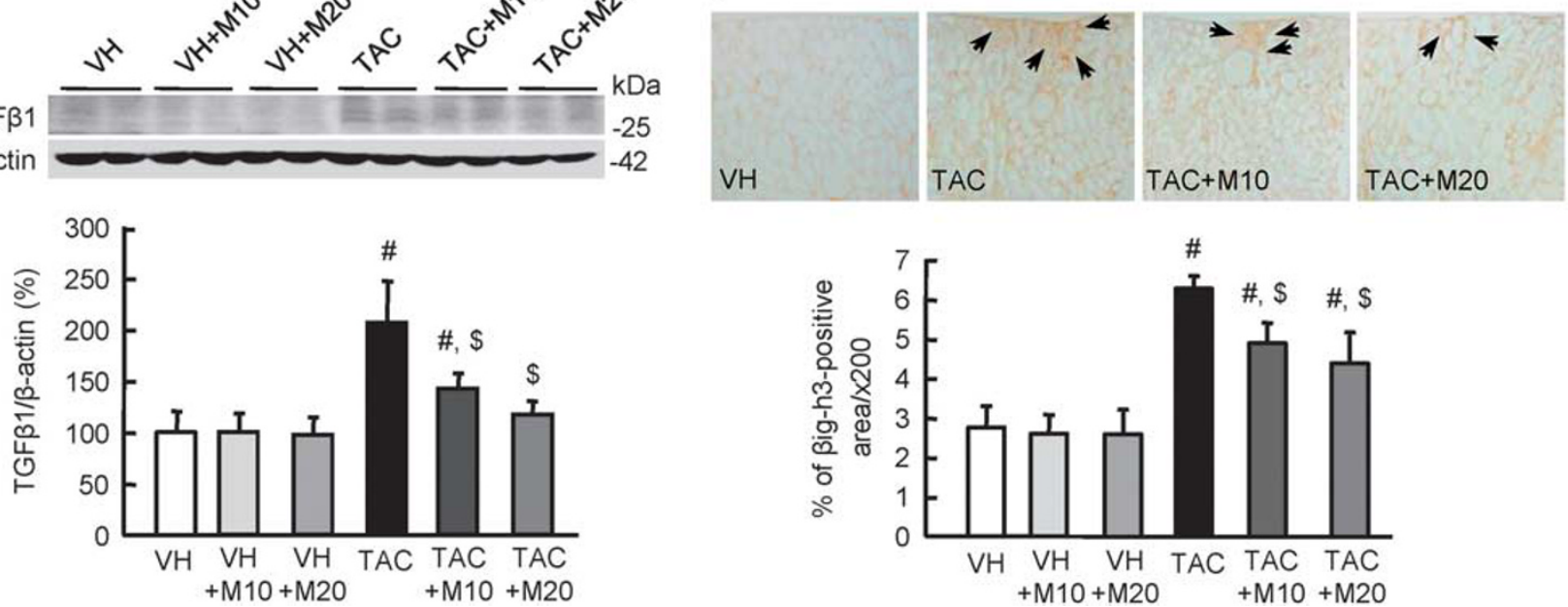

Figure 2 Effect of MK0626 (M) on fibrosis during tacrolimus (TAC)-induced renal injury. (a and b) Histological analysis in the renal cortex treated with TAC for 4 weeks shows striped tubulointerstitial fibrosis, mononuclear cell infiltration, and tubular atrophy. MK0626 treatment significantly reduces these damages compared with TAC treatment. (c-e) Immunoblot analysis of e-cadherin, $\alpha$-smooth muscle actin (a-SMA), and transforming growth factor $\beta 1$ (TGF $\beta 1$ ) in the renal cortex. The relative optical densities of bands in each lane were normalized to each $\beta$-actin band from the same gel. (f) Immunohistochemistry and quantitative graph for the TGF $\beta 1$-inducible gene-h3 ( $\beta$ ig-h3) in the renal cortex. Note that the expression of e-cadherin is recovered and that $\alpha$-SMA, TGF $\beta 1$, and $\beta$ ig-h3 are decreased after combined treatment with MK0626. The arrows indicate $\beta$ ig-h3-positive areas. Magnification, $\times 400 . n=8$ per group. ${ }^{\#} P<0.05$ vs $\mathrm{VH}^{;}{ }^{\$} P<0.05$ vs TAC. 
a
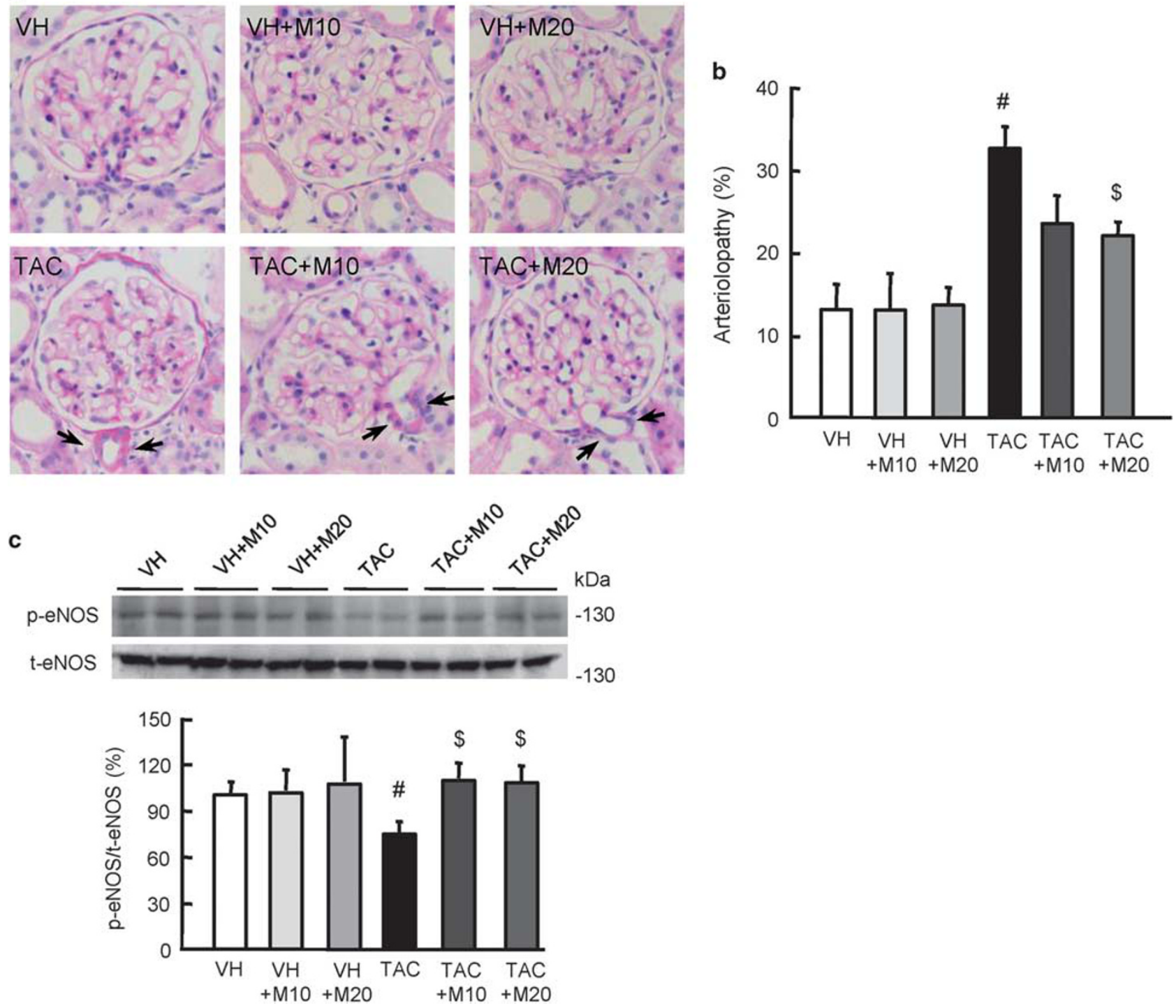

Figure 3 Effect of MK0626 (M) on tacrolimus (TAC)-induced arteriolopathy and expression of eNOS. (a and b) Periodic acid-Schiff (PAS) staining in tissue sections and its quantitative analysis. The TAC group shows a typical afferent arteriolopathy, as illustrated by a glomerulus with expansion of the cytoplasm of terminal arteriolar smooth muscle cells by eosinophilic, granular material. (c) Immunoblot analysis of phosphorylated endothelial nitric oxide synthase (p-eNOS) and total eNOS (t-eNOS). The relative optical densities of bands in each lane were normalized to each $\beta$-actin band from the same gel. Note that the MK0626 group shows reduced arteriolopathy and expression of renin and increased levels of eNOS. The arrows indicate PASpositive afferent arterioles. Magnification, $\times 400 . n=8$ per group. ${ }^{\#} P<0.05 \mathrm{vs} \mathrm{VH} ;{ }^{\$} P<0.05$ vs TAC.

groups. The strong nuclear expression and concentration in the urine of 8-OHdG were markedly increased in the TAC group, and these effects were reversed after MK0626 treatment. We further evaluated whether MK0626 affects the expression of the antioxidative-stress-related molecules MnSOD, HO-1 (inducible form), and HO-2 (constitutive form) and expressed this effect as the ratio of HO-1/HO-2. The expression of these molecules was reduced in the TAC group compared with the $\mathrm{VH}-$ or $\mathrm{VH}+\mathrm{M}$-treated groups and was recovered by MK0626 treatment.

\section{Effect of MK0626 on TAC-Induced Apoptosis}

Next we evaluated whether MK0626 treatment affects apoptosis, which is an important mechanism of cell death in TAC-induced renal injury. The number of TUNEL-positive cells in tissue sections was significantly higher in the TAC group compared with the $\mathrm{VH}$ group, and this was reduced by the addition of MK0626 (Figures 5a and b). Furthermore, TAC treatment suppressed the expression of Bcl-2, which was recovered by MK0626 administration. In contrast, the induction of Bax, Bim, and active caspase- 3 by TAC was attenuated by the addition of MK0626 (Figures 5c-f). 


\section{Activity of DPP IV and Substrates of DPP IV in Experimental Animals}

A 4-week TAC treatment significantly increased plasma and renal cortex DPP IV activity, which was effectively suppressed by MK0626 treatment, as shown in Figures $6 \mathrm{a}$ and $\mathrm{b}$. The substrates of DPP IV, including GLP-1, NPY, and SDF-1, were measured in the blood using ELISA. The level of active GLP-1 was markedly suppressed in the TAC group, and this was restored after combined treatment with MK0626, in a dose-dependent manner (Figure 6c). In contrast with GLP-1, the level of NPY and SDF-1 did not vary among the experimental groups (Figures $6 \mathrm{~d}$ and e). Based on this a
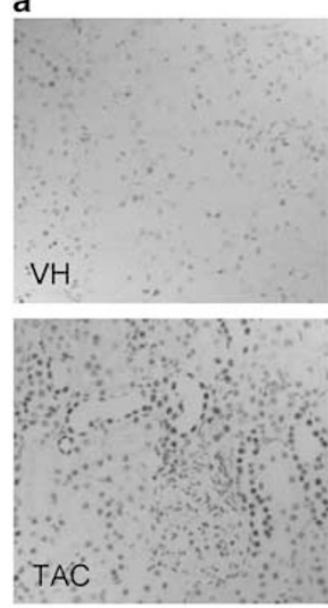
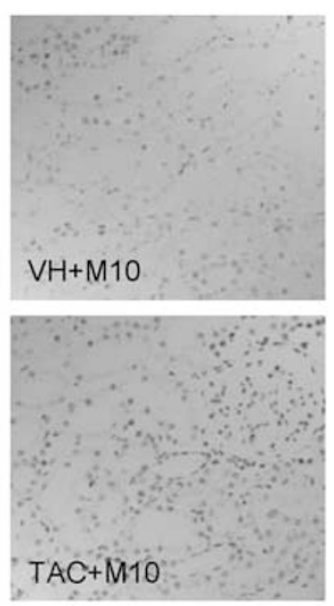
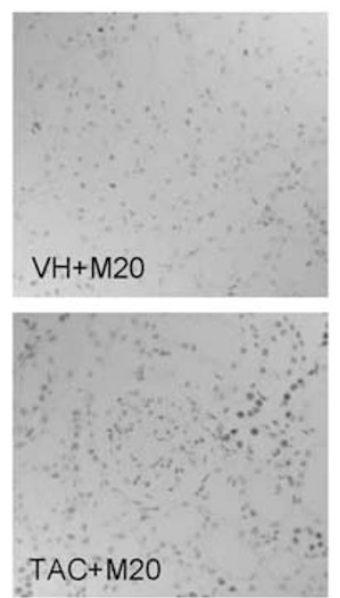

b

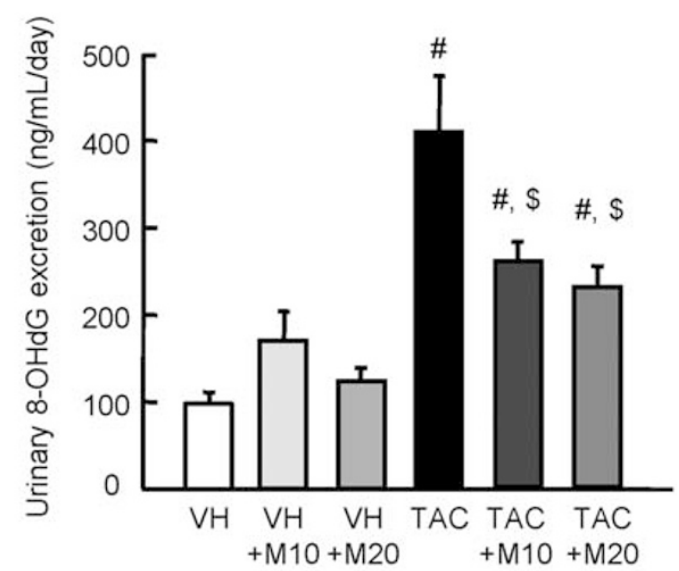

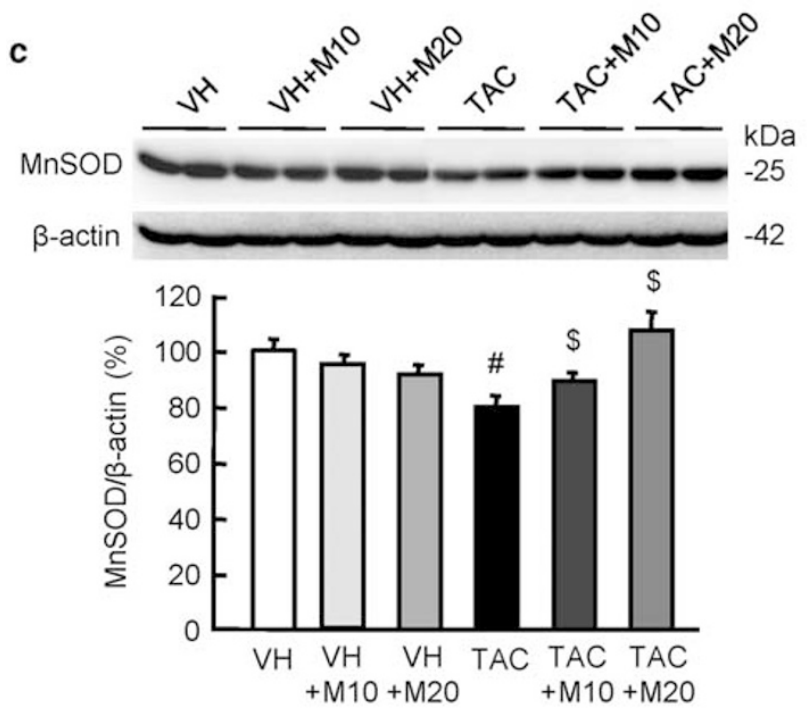
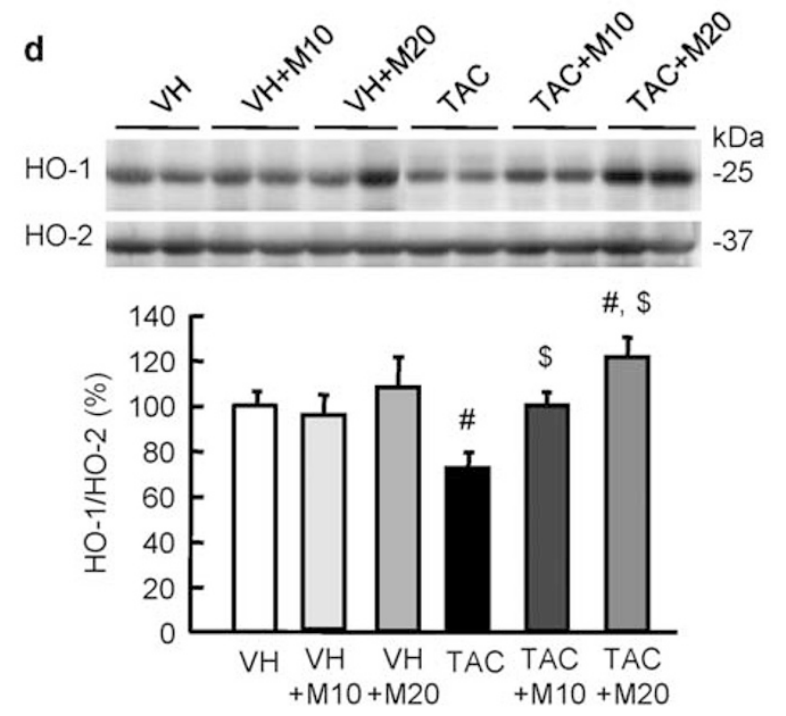

Figure 4 Effect of MK0626 (M) on oxidative stress during tacrolimus (TAC)-induced renal injury. (a) Immunohistochemistry for 8-hydroxy-2'deoxyguanosine (8-OHdG) in tissue sections. Intense nuclear expression of 8-OHdG is shown in the TAC group, and the administration of MK0626 reduces its expression levels. (b) Urinary 8-OHdG excretion per day. The TAC-induced 8-OHdG excretion is lowered by the coadministration of MK0626. (c and d) Immunoblot analysis of manganese superoxide dismutase (MnSOD), heme oxygenase-1 (HO-1, inducible form), and HO-2 (constitutive form) in the renal cortex from the experimental animals. The relative optical densities of bands in each lane were normalized to each $\beta$-actin band from the same gel. Note that combined treatment with MK0626 restored their expression compared with TAC. Magnification, $\times 400$. $n=8$ per group. ${ }^{\#} P<0.05$ vs $\mathrm{VH} ;{ }^{\$} \mathrm{P}<0.05$ vs TAC.

Figure 5 Effect of MK0626 (M) on apoptosis during tacrolimus (TAC)-induced renal injury. (a and b) In situ TdT-mediated dUTP-biotin nick end labeling (TUNEL) assay and its analysis to detect apoptosis in the renal tissue sections of the experimental groups. MK0626 treatment significantly reduces TUNEL-positive apoptotic bodies compared with TAC. (c-f) Immunoblot analysis of Bcl-2, Bax, Bim, and active caspase-3 in the renal cortex. Note that MK0626 treatment significantly recovers the expression of $\mathrm{Bcl}-2$ and downregulates Bax, Bim, and active caspase-3 compared with TAC. The relative optical densities of bands in each lane were normalized to each $\beta$-actin band from the same gel. The arrows indicate TUNEL-positive apoptotic bodies. Magnification, $\times 400 . n=8$ per group. ${ }^{*} P<0.05$ vs $\mathrm{VH} ;{ }^{\$} P<0.05$ vs TAC. 
a
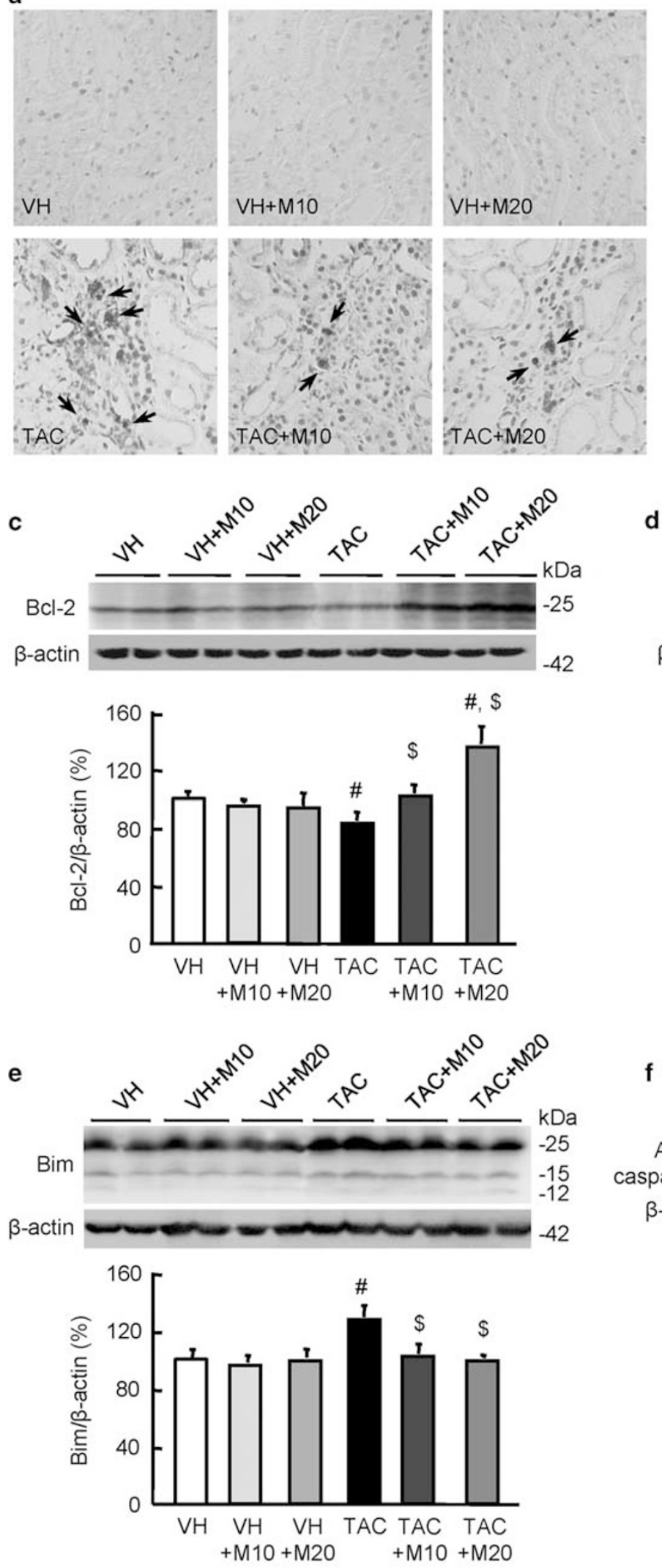

b

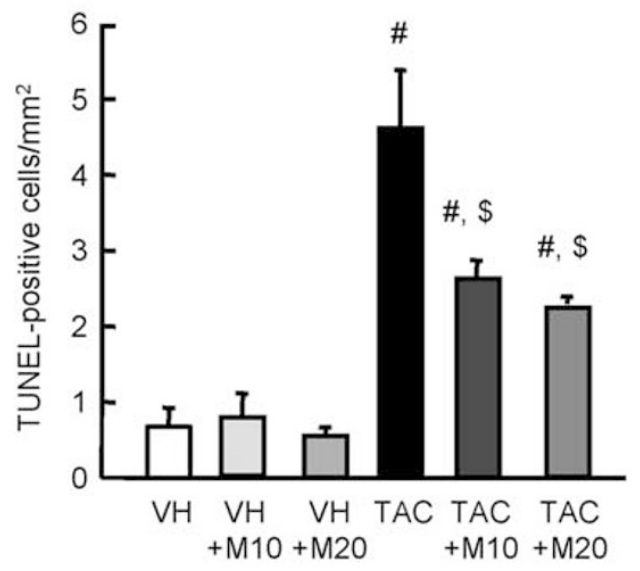

d
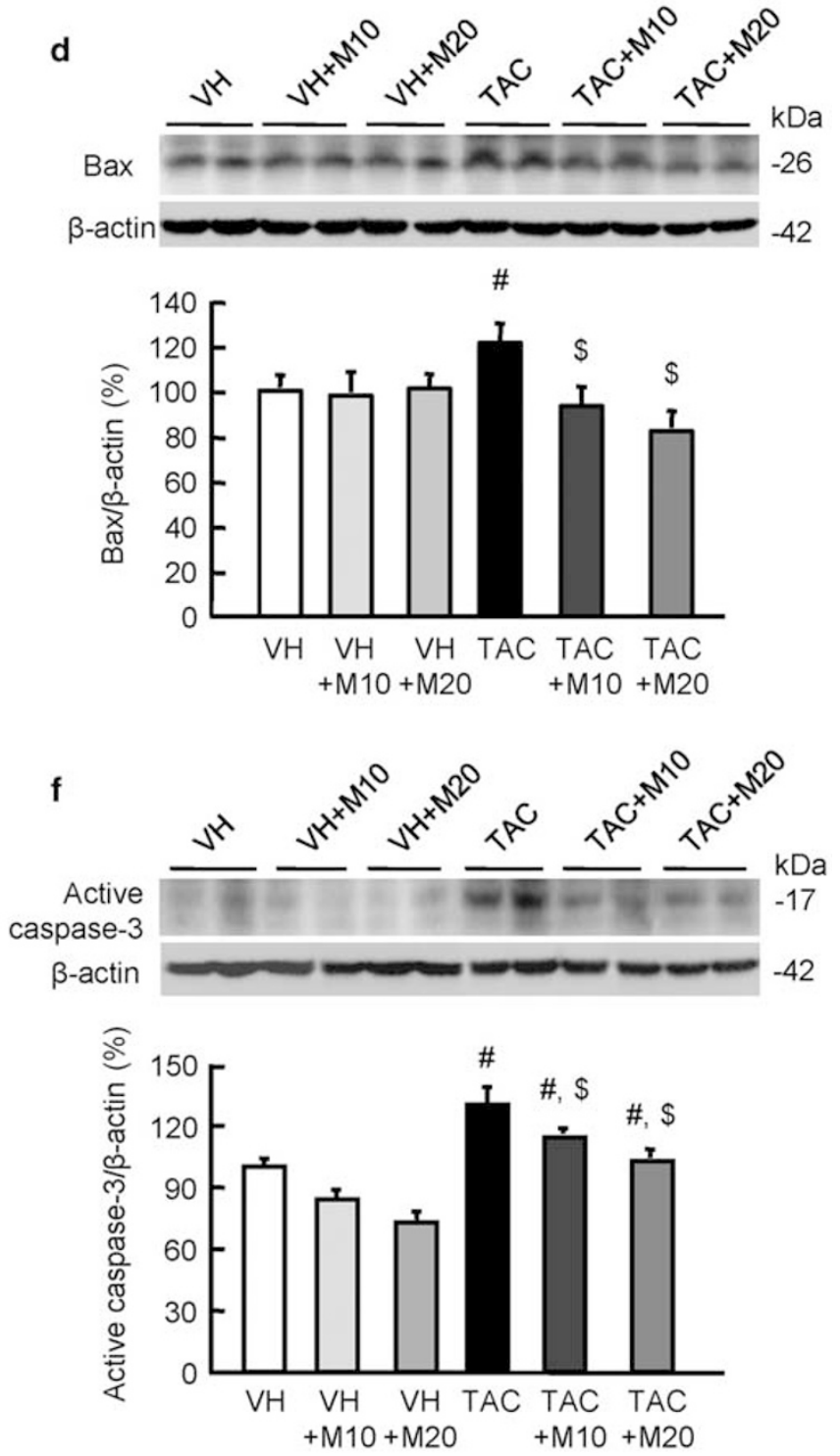

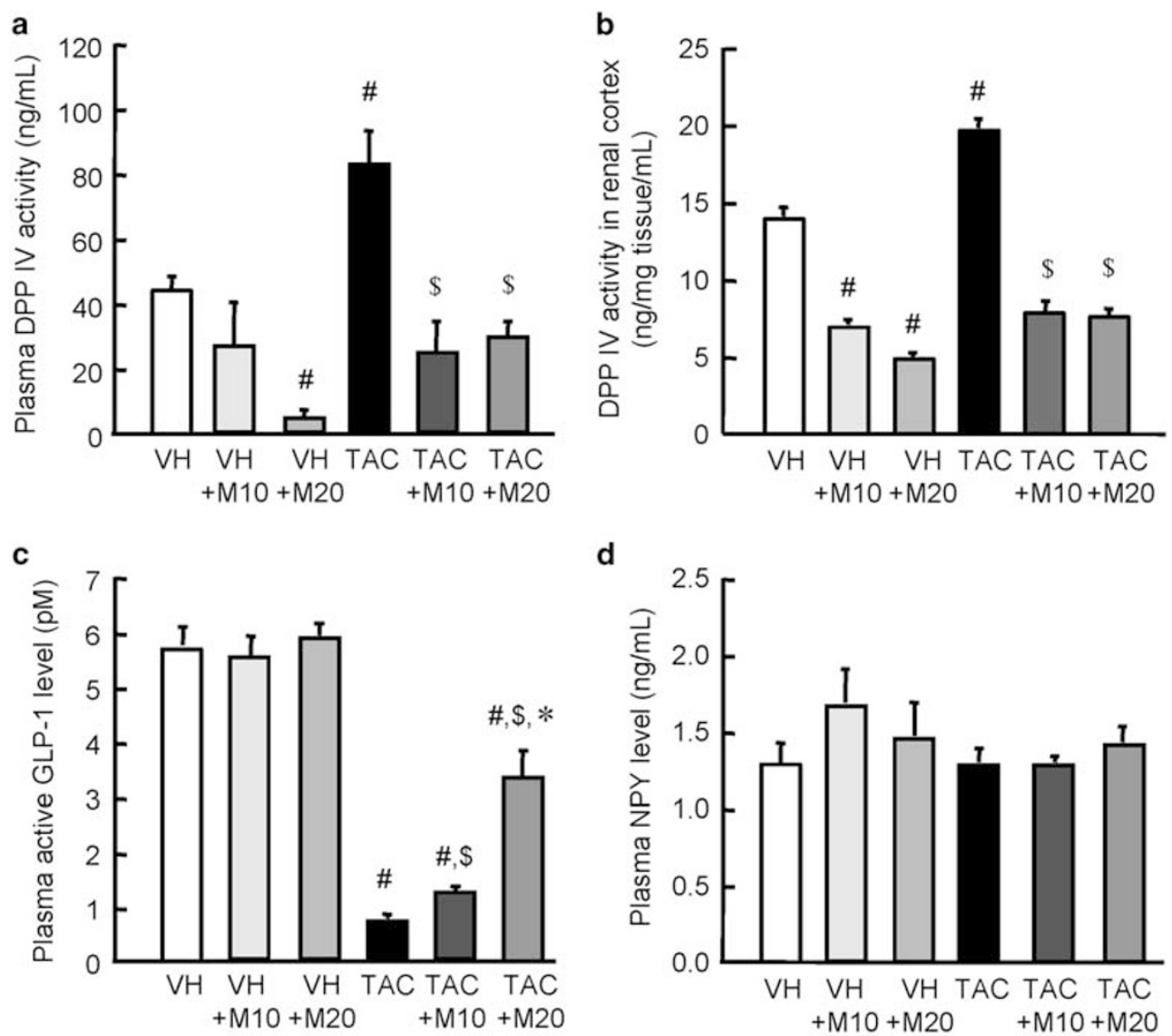

d
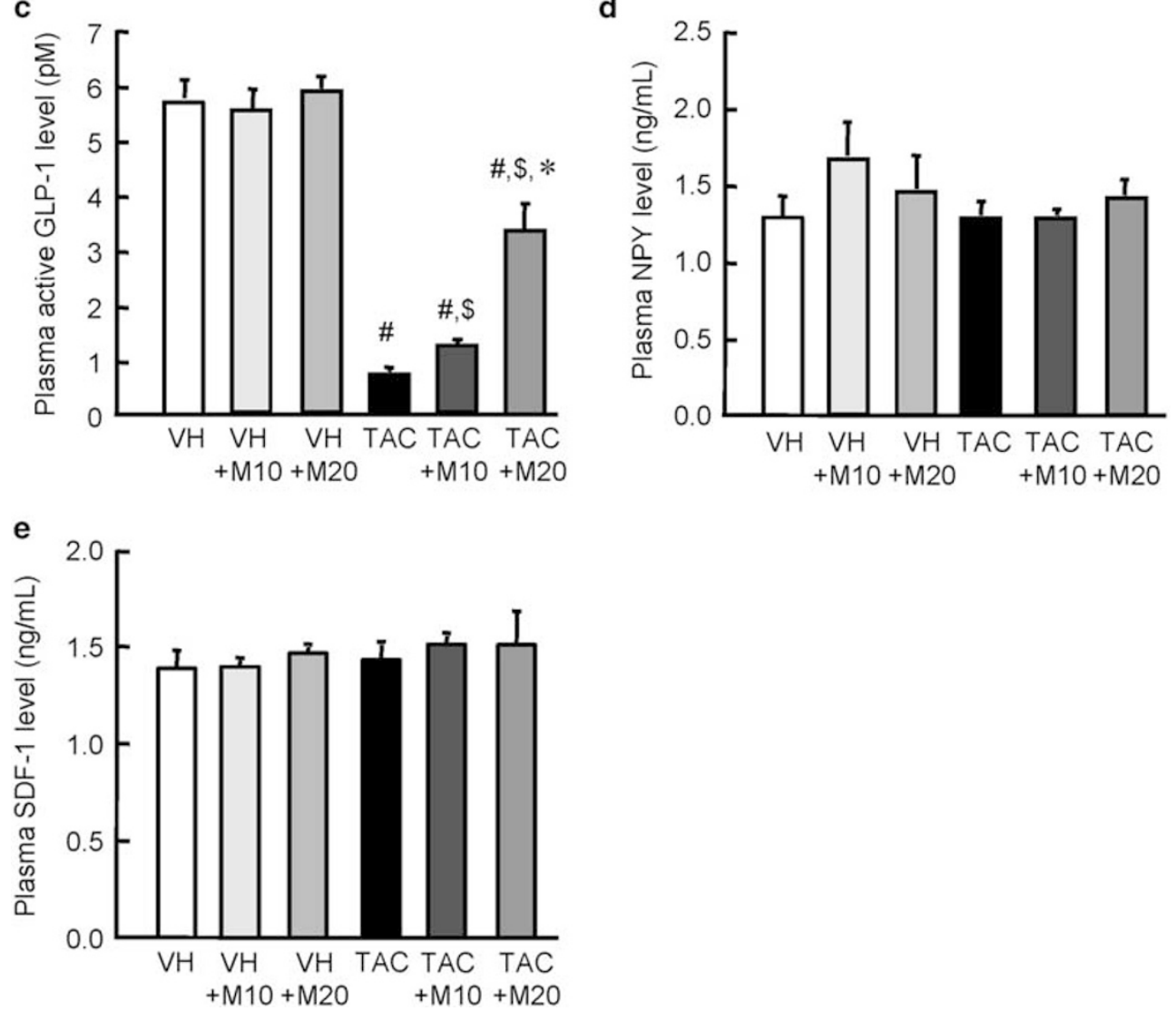

Figure 6 Plasma and renal DPP IV activity and levels of the plasma glucagon-like peptide-1 (GLP-1), neuropeptide Y (NPY), and stromal cell-derived factor-1 (SDF-1) in experimental animals. (a and b) DPP IV activity is evaluated in the plasma and renal cortex of experimental animals. DPP IV activity is suppressed significantly by MK0626 (M). (c) The level of bioactive GLP-1 is remarkably higher in the MK0626 and tacrolimus (TAC) groups. (d and e) The other DPP IV substrates, NPY, and SDF-1 are also evaluated. The plasma NPY and SDF-1 levels are not different among the experimental groups. $n=8$ per group. ${ }^{\#} P<0.05$ vs $\mathrm{VH} ;{ }^{\$} P<0.05$ vs $\mathrm{TAC} ;{ }^{*} P<0.05$ vs $\mathrm{TAC}+\mathrm{M} 10$.

finding, we examined whether the GLP-1 signaling pathway is associated with the protective effect of MK0626. We performed immunohistochemical and immunoblot analyses of the GLP-1R. Abundant expression of GLP-1R was observed in the apical membrane and cytosol of proximal tubules (PTs) in the $\mathrm{VH}$ group; in contrast, its expression was reduced in the damaged PTs in the TAC group. Combined treatment with MK0626 led to the recovery of the immunoreactivity and protein amount of GLP-1R, as shown in Figure 7.

\section{DISCUSSION}

The present study was undertaken to investigate whether the DPP IV inhibitor MK0626 has a protective effect against TAC-induced nephropathy. MK0626 attenuated renal 

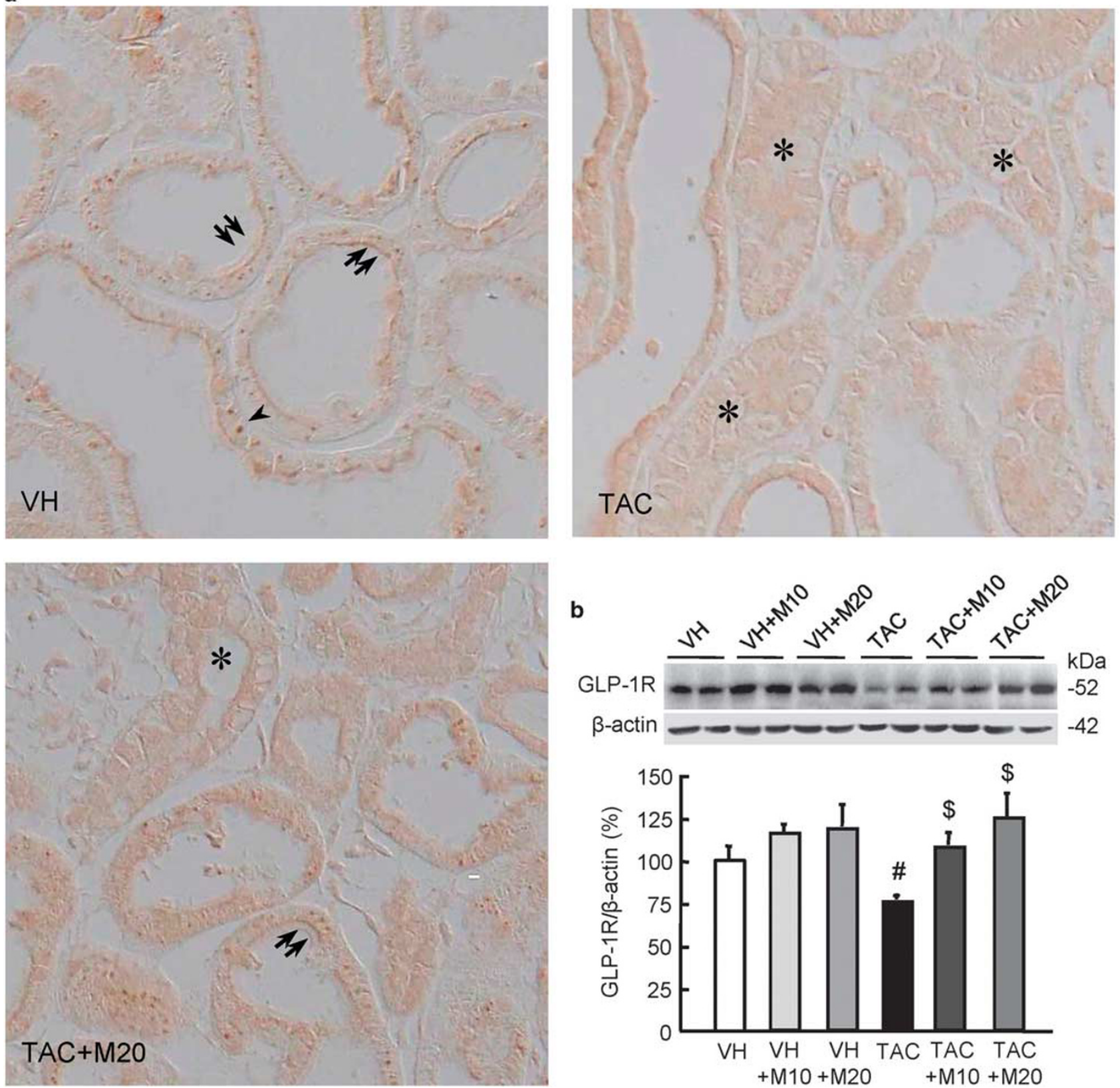

Figure 7 Effect of MK0626 (M) on the glucagon-like peptide-1 receptor (GLP-1R) during tacrolimus (TAC)-induced renal injury. (a) Immunoreactivity for GLP-1R is mainly localized in the apical membrane and cytosol of proximal tubules (PTs) in the vehicle (VH) group. The TAC group shows markedly reduced expression in damaged PTs. Note that combined treatment with MK0626 restored their expression compared with TAC treatment. (b) The reduced amount of the GLP-1R protein is significantly restored by MK0626, which was confirmed by immunoblot analysis. The relative optical densities of bands in each lane were normalized to each $\beta$-actin band from the same gel. The arrowhead indicates GLP-1R-positive cytoplasm; double arrows indicate GLP-1R-positive apical membranes; asterisks indicate GLP-1R-negative PTs. Magnification, $\times 400 . n=8$ per group. ${ }^{\#} P<0.05$ vs VH; ${ }^{\$} P<0.05$ vs TAC.

dysfunction with histological improvement in TAC-treated rats by reducing oxidative stress and apoptosis. In addition, we found that the increased DPP IV activity observed after TAC administration was effectively inhibited by treatment with MK0626. Interestingly, among the various substrates of DPP IV, GLP-1 and its receptor (but not NYP and SDF-1) responded to the changes in DPP IV activity. These findings suggest that the DPP IV inhibitor MK0626 has beneficial properties by exerting antioxidative and antiapoptotic effects via enhanced GLP-1 signaling in TAC-induced nephropathy.

We showed that a decrease in inflammation ameliorates calcineurin inhibitor (CNI)-induced toxicity, ${ }^{18,19,21,22}$ suggesting that TAC treatment itself recruits inflammatory cells and proinflammatory cytokines. Practically, depletion of 
macrophages using liposome-encapsulated clodronate significantly decreased CsA-induced renal dysfunction and fibrosis. ${ }^{23}$ Therefore, we investigated whether DPP IV inhibition affects TAC-induced macrophage infiltration and inflammatory factors. Many studies have demonstrated that DPP IV inhibitors inhibit the production of inflammatory cytokines and the activation of the nuclear factor- $\kappa \mathrm{B}$, suggesting that it has an anti-inflammatory effect. ${ }^{10,12,24}$ Consistent with this, the current in vivo results showed that the administration of MK0626 reduced the number of positive cells and the immunoreactivity of ED-1 in the injured area, as well as the levels of OPN, as shown in Figure 1. These findings suggest that MK0626 exerts an antiinflammatory effect via the inhibition of macrophage migration to the injured area, thus resulting in a reduction in the production of proinflammatory cytokines.

Chronic TAC nephrotoxicity in humans is characterized by typical histological features, such as striped interstitial fibrosis. This contributes to the annual risk of end-stage renal disease and increases over time in human recipients of renal transplants. ${ }^{25}$ We and others have demonstrated that TGF $\beta 1$ is mainly involved in TAC-induced fibrosis. ${ }^{1,3}$ In fact, the administration of a neutralizing anti-TGF $\beta 1$ antibody abrogates CsA-associated structural damage. ${ }^{26}$ We expected that MK0626 would protect against the progression of TACinduced renal fibrosis, because the antifibrotic effect of MK0626 was shown in an animal model of 5/6 nephrectomy and unilateral ureteral obstruction. ${ }^{10,27}$ Our results demonstrated that cotreatment with MK0626 diminished the expression of TGF $\beta 1$, its bioactive form $\beta$ ig-h3, and $\alpha$-SMA and recovered the expression of e-cadherin, which is a cellcell adhesion molecule; these molecular effects were accompanied by a significant attenuation of renal fibrosis. Thus our findings suggest that MK0626 effectively suppresses the progression of inflammation and fibrosis in TAC-induced nephrotoxicity.

Oxidative stress caused by reactive oxygen species (ROS) is a common pathway of CNI-induced renal injury. ${ }^{28}$ The high level of oxidative stress induced by chronic TAC administration causes structural and functional kidney impairment, because short- and long-term TAC treatment induces the production of free radical species in the kidney, causing apoptotic cell death. In this study, we evaluated whether MK0626 affects TAC-induced oxidative stress and apoptosis. Our results showed that MK0626 not only dramatically recovered MnSOD and $\mathrm{HO}-1$ expression but also reduced the number of TUNEL-positive cells. In fact, there is increasing evidence of an association between the development of renal damage and increased oxidative stress, and of the function of DPP IV inhibitors as scavengers of ROS, with a resultant improvement of renal function and histopathology. ${ }^{29,30}$ Taken together, these findings suggest that MK0626 protects against TAC-induced renal injury via a decrease in oxidative stress and subsequent apoptotic cell death.
In the present study, we found that TAC group showed higher DPP IV activity than that of the VH group. The reason for increase in DPP IV in our model is unclear, but we can speculate several possibility. First, increased insulin sensitivity may associate with DPP IV activity in our model. It is well known that DPP IV activity is associated with insulin resistance in Types 1 and 2 diabetic patients, and it might have an important role in its pathophysiology. ${ }^{31}$ In addition, several reports proposed that hypoxia may upregulate DPP IV activity, and it was detected as a novel target gene of the hypoxia-induced factor- $1 .{ }^{32,33}$ Others revealed that the exogenous administration of TGF $\beta 1$, which is a well-known central player in renal fibrosis, markedly enhanced DPP IV activity in proximal tubules cells. ${ }^{27}$ Based on the findings described above, we propose that TAC-induced low-grade ischemia and TGF $\beta 1$ activation in this study are also causative factors of TAC-induced DPP IV activity. However, further understanding of the exact mechanism underlying DPP IV in chronic TAC nephropathy is required.

Next we investigated which substrates of DPP IV are involved in the protective effect observed in this experiment. In fact, most studies have focused on the effect of GLP-1, because the DPP IV inhibitor was developed as an antidiabetic agent to increase the bioavailability of GLP-1. Because other substrates, such as NPY, SDF-1, etc, also have cell-protective properties, ${ }^{34-37}$ the present study was undertaken to investigate which substrates are associated with the protective effect of the DPP IV inhibitor against TAC-induced renal damage. The results showed that active GLP-1 was suppressed in response to the highly activated DPP IV, which was recovered by treatment with MK0626 in a dose-dependent manner (Figure 6b). In contrast to GLP-1 excursion, the levels of NPY and SDF-1 did not change among the experimental groups. Furthermore, the reduced renal expression of GLP-1R observed after TAC administration was also increased by treatment with MK0626. This observation is consistent with recent reports that GLP-1 contributes to the antiapoptotic effect of DPP IV inhibitors in cisplatin-induced renal injury, whereas NPY and SDF-1 do not contribute to renal protection. Taken together, these findings suggest that DPP IV inhibition decreases TAC-induced oxidative stress and subsequent apoptotic cell death via enhanced GLP-1 signaling.

The experimental model of chronic TAC nephropathy has some limitations. Unlike humans, rodents are resistance to TAC-induced renal injury. Therefore, we used a low-salt diet to induce the functional impairment and morphological consequences of TAC similar to humans. A low-salt diet was used to activate the renin-angiotensin system (RAS), because activation of RAS is important in the pathogenesis of TACinduced renal injury. ${ }^{1,28,38}$

In conclusion, our results suggest that DPP IV inhibition is effective in improving TAC-induced nephropathy via the preservation of GLP-1 signaling, independently of its hypoglycemic effects. Therefore, a targeted therapy aimed at inhibiting DPP IV may be a useful new approach in the 
management of the progression of nephropathy in renaltransplant patients receiving TAC.

\section{ACKNOWLEDGMENTS}

We thank Merck Sharp and Dohme, New Jersey, USA for kindly providing MK-0626. This work was supported by a Basic Science Research Program through the National Research Foundation of Korea (NRF) funded by the Ministry of Science, ICT and Future Planning (2012R1A1A3001890 and 2015R1A1A3A04000946) and the Korean Health Technology R\&D Project, Ministry for Health and Welfare, Republic of Korea (HI04C3417).

\section{DISCLOSURE/CONFLICT OF INTEREST}

The authors declare no conflict of interest.

1. Shihab FS, Bennett WM, Tanner AM et al. Mechanism of fibrosis in experimental tacrolimus nephrotoxicity. Transplantation 1997;64: 1829-1837.

2. Han D, Piao S, Song J-H et al. Effect of sirolimus on calcineurin inhibitor-induced nephrotoxicity using renal expression of KLOTHO, an antiaging gene. Transplantation 2010;90:135-141.

3. Hwang $\mathrm{H}$, Ghee JY, Song $\mathrm{JH}$ et al. Comparison of adverse drug reaction profiles of two tacrolimus formulations in rats. Immunopharmacol Immunotoxicol 2012;34:434-442.

4. Piao S, Lim S, Doh K et al. Combined treatment of tacrolimus and everolimus increases oxidative stress by pharmacological interactions. Transplantation 2014;98:22-28.

5. Neumiller J, Wood L, Campbell RK. Dipeptidyl peptidase-4 inhibitors for the treatment of type 2 diabetes mellitus. Pharmacotherapy 2010;30: 463-484.

6. Shih C-M, Chen Y-H, Lin Y-W et al. MK-0626, a dipeptidyl peptidase-4 inhibitor, improves neovascularization by increasing both the number of circulating endothelial progenitor cells and endothelial nitric oxide synthetase expression. Curr Med Chem 2014;21:2012-2022.

7. Azuma K, Radikova Z, Mancino J et al. Measurements of islet function and glucose metabolism with the dipeptidyl peptidase 4 inhibitor vildagliptin in patients with type 2 diabetes. J Clin Endocrinol Metab 2008;93:459-464.

8. Campbell RK, White J. More choices than ever before: emerging therapies for type 2 diabetes. Diabetes Educ 2008;34:518-534.

9. Glorie LL, Verhulst A, Matheeussen V et al. DPP4 inhibition improves functional outcome after renal ischemia-reperfusion injury. Am J Physiol Renal Physiol 2012;303:F681-F688.

10. Joo KW, Kim S, Ahn SY et al. Dipeptidyl peptidase IV inhibitor attenuates kidney injury in rat remnant kidney. BMC Nephrol 2013;14: 98.

11. Katagiri D, Hamasaki Y, Doi K et al. Protection of glucagon-like peptide1 in cisplatin-induced renal injury elucidates gut-kidney connection. J Am Soc Nephrol 2013;24:2034-2043.

12. Park $\mathrm{C}, \mathrm{Kim} \mathrm{H}, \mathrm{Ko} \mathrm{S}$ et al. Long-term treatment of glucagon-like peptide-1 analog exendin-4 ameliorates diabetic nephropathy through improving metabolic anomalies in $\mathrm{db} / \mathrm{db}$ mice. J Am Soc Nephrol 2007;18:1227-1238.

13. Jin L, Lim S, Doh K et al. Dipeptidyl peptidase IV inhibitor MK-0626 attenuates pancreatic islet injury in tacrolimus-induced diabetic rats. PLoS One 2014;9:e100798-e100798.

14. Lotfy $M$, Singh J, Rashed $\mathrm{H}$ et al. Mechanism of the beneficial and protective effects of exenatide in diabetic rats. J Endocrinol 2014;220: 291-304.

15. Piao S, Bae S, Lim S et al. Drug interaction between cyclosporine and mTOR inhibitors in experimental model of chronic cyclosporine nephrotoxicity and pancreatic islet dysfunction. Transplantation 2012;93:383-389.

16. Li C, Yang CW, Park JH et al. Pravastatin treatment attenuates interstitial inflammation and fibrosis in a rat model of chronic cyclosporine-induced nephropathy. Am J Physiol Renal Physiol 2004;286:F46-F57.

17. Lim S, Hyoung B, Piao S et al. Chronic cyclosporine nephropathy is characterized by excessive autophagosome formation and decreased autophagic clearance. Transplantation 2012;94:218-225.

18. Doh KC, Lim SW, Piao SG et al. Ginseng treatment attenuates chronic cyclosporine nephropathy via reducing oxidative stress in an experimental mouse model. Am J Nephrol 2013;37:421-433.

19. Lim $S$, Doh $K$, Jin $L$ et al. Oral administration of ginseng ameliorates cyclosporine-induced pancreatic injury in an experimental mouse model. PLoS One 2013;8:e72685-e72685.

20. Lim SW, Doh KC, Jin L et al. Ginseng treatment attenuates autophagic cell death in chronic cyclosporine nephropathy. Nephrology (Carlton) 2014;19:490-499.

21. Kim SH, Ghee JY, Piao S et al. Early and delayed effects of AST-120 on chronic cyclosporine nephropathy. Nephrol Dial Transplant 2011;26: 1502-1515.

22. Piao S, Kang S, Lim S et al. Influence of N-acetylcysteine on Klotho expression and its signaling pathway in experimental model of chronic cyclosporine nephropathy in mice. Transplantation 2013;96: 146-153.

23. Ghee JY, Han DH, Song HK et al. The role of macrophage in the pathogenesis of chronic cyclosporine-induced nephropathy. Nephrol Dial Transplant 2008;23:4061-4069.

24. Marques C, Mega C, Goncalves A et al. Sitagliptin prevents inflammation and apoptotic cell death in the kidney of type 2 diabetic animals. Mediators Inflam. 2014;2014:538737.

25. de Mattos AM, Olyaei AJ, Bennett WM. Nephrotoxicity of immunosuppressive drugs: long-term consequences and challenges for the future. Am J Kidney Dis 2000;35:333-346.

26. Islam M, Burke Jr. JF , McGowan TA et al. Effect of anti-transforming growth factor-beta antibodies in cyclosporine-induced renal dysfunction. Kidney Int 2001;59:498-506.

27. Min HS, Kim JE, Lee MH et al. Dipeptidyl peptidase IV inhibitor protects against renal interstitial fibrosis in a mouse model of ureteral obstruction. Lab Invest 2014;94:598-607.

28. Yoon HE, Yang CW. Established and newly proposed mechanisms of chronic cyclosporine nephropathy. Korean J Intern Med 2009;24:81-92.

29. Vaghasiya J, Sheth N, Bhalodia $\mathrm{Y}$ et al. Sitagliptin protects renal ischemia reperfusion induced renal damage in diabetes. Regul Pept 2011;166:48-54.

30. Chen YT, Tsai TH, Yang CC et al. Exendin-4 and sitagliptin protect kidney from ischemia-reperfusion injury through suppressing oxidative stress and inflammatory reaction. J Transl Med 2013;11:270.

31. Blaslov K, Bulum T, Duvnjak L. Circulating dipeptidyl peptidase-4 activity is associated with insulin resistance in type 1 diabetic patients. J Diabetes Complications 2015;29:390-394.

32. Eltzschig $\mathrm{H}$, Faigle $\mathrm{M}$, Knapp $\mathrm{S}$ et al. Endothelial catabolism of extracellular adenosine during hypoxia: the role of surface adenosine deaminase and CD26. Blood 2006;108:1602-1610.

33. Dang D, Chun S, Burkitt K et al. Hypoxia-inducible factor- 1 target genes as indicators of tumor vessel response to vascular endothelial growth factor inhibition. Cancer Res 2008;68:1872-1880.

34. Drucker DJ. The biology of incretin hormones. Cell Metab 2006;3:153-165.

35. Drucker DJ. Dipeptidyl peptidase-4 inhibition and the treatment of type 2 diabetes: preclinical biology and mechanisms of action. Diabetes Care 2007;30:1335-1343.

36. Stokman G, Stroo I, Claessen N et al. SDF-1 provides morphological and functional protection against renal ischaemia/reperfusion injury. Nephrol Dial Transplant 2010;25:3852-3859.

37. Liu L, Xie S, Liao $X$ et al. Netrin-1 pretreatment protects rat kidney against ischemia/reperfusion injury via suppression of oxidative stress and neuropeptide $\mathrm{Y}$ expression. J Biochem Mol Toxicol 2013;27: 231-236.

38. Stillman IE, Andoh TF, Burdmann EA et al. FK506 nephrotoxicity: morphologic and physiologic characterization of a rat model. Lab Invest 1995;73:794-803. 